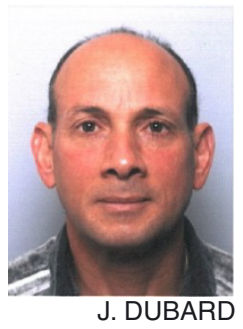

\title{
Qualification métrologique de spectrophotomètre : un guide pour les industriels
}

\section{Characterization of spectrophotometer: guide for industry}

\author{
Jimmy DUBARD, Jean VOYER, Jacques HAMEURY et François BUTEAU \\ Laboratoire national de métrologie et d'essais (LNE), 29 avenue Roger Hennequin, 78197 Trappes Cedex, France, jimmy.dubard@lne.fr.
}

\begin{abstract}
Résumé
Les spectrophotomètres permettent de mesurer les propriétés optiques des matériaux telles la transmission ou la réflexion. Ils sont utilisés dans de nombreux domaines industriels tels que la santé, le transport, l'énergie... Pour assurer la traçabilité des mesures à travers le raccordement des étalons de transmission ou de réflexion le LNE dispose de spectrophotomètres qui font l'objet d'une qualification métrologique tout au long de la durée de vie de ces équipements. Nous présentons les étapes de cette qualification. Les résultats sont utilisés pour évaluer les incertitudes de mesure. La procédure suivie peut servir de guide pour les utilisateurs de spectrophotomètre désirant évaluer les incertitudes avec lesquelles ils réalisent leurs mesures.
\end{abstract}

MOTS CLÉS : SPECTROPHOTOMÈTRE, TRANSMISSION, QUALIFICATION, INCERTITUDE DE MESURE.

\begin{abstract}
Spectrophotometers allow measurement of the optical properties of materials such as transmittance or reflectance. They are used in many industrial fields: health, transport, energy... To ensure the traceability of the measurements through the calibration of transmittance and reflectance standards, the LNE uses spectrophotometers that are qualified along the lifetime of these instruments. We present the qualification steps. The results are used to evaluate the uncertainties. The procedure followed can be used as a guideline for spectrophotometer users who want to evaluate their measurement uncertainties.
\end{abstract}

KEY WORDS: SPECTROPHOTOMETER,

TRANSMITTANCE, QUALIFICATION, MEASUREMENT UNCERTAINTY.

\section{Introduction}

Les spectrophotomètres sont des appareils couramment utilisés dans des laboratoires d'analyses médicales ou des laboratoires de caractérisation de matériaux [1-3].
Ils permettent de mesurer des paramètres optiques tels que la transmission et la réflexion sur un domaine spectral pouvant comprendre l'ultraviolet (UV), le visible (vis.) et l'infrarouge (IR). Les mesures réalisées doivent être traçables et fiables. Ceci est obtenu en effectuant une caractérisation complète des performances de ces appareils de mesure.

Les appareils sont plus ou moins complexes et font intervenir des techniques de mesure différentes : simple ou double faisceaux, faisceau libre, sphère intégrante, accessoire VW... Leur qualification doit être réalisée selon une procédure maîtrisée. Cet article traite de la qualification des spectrophotomètres couramment utilisés dans l'industrie.

Nous présentons tout d'abord le principe de fonctionnement d'un spectrophotomètre et les différentes étapes de la qualification qui comprend une caractérisation du spectrophotomètre selon les deux paramètres du résultat d'une mesure : longueur d'onde et amplitude. Puis nous utilisons les résultats de cette qualification pour évaluer les incertitudes de mesure.

\section{Présentation d'un spectrophotomètre}

Un spectrophotomètre permet de réaliser des mesures des propriétés optiques spectrales de matériaux. Les éléments principaux constituant un spectrophotomètre sont (fig. 1):

- un générateur de faisceaux de lumière monochromatique comprenant une ou deux sources 


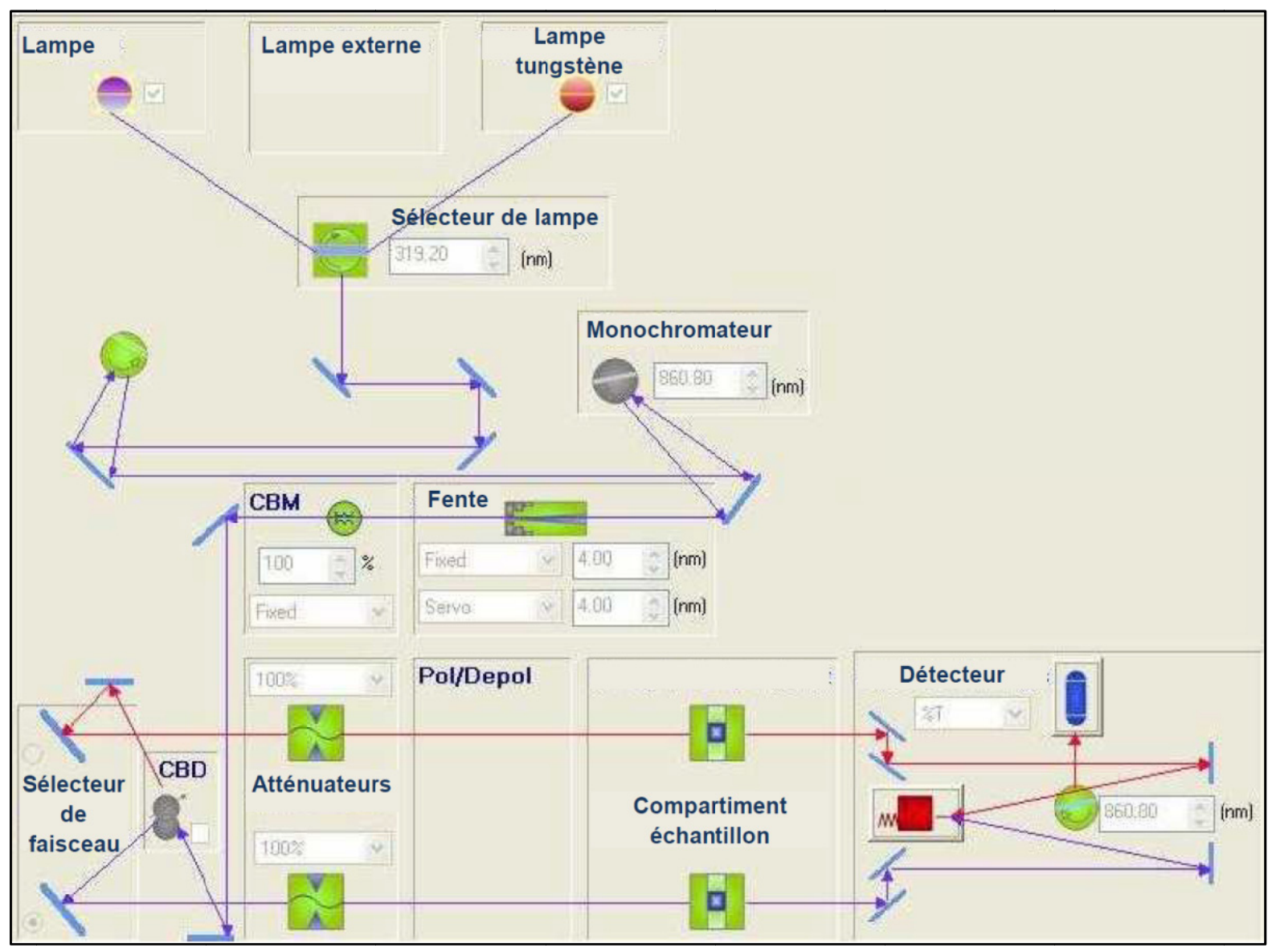

Fig. 1. - Schéma de principe d'un spectrophotomètre fonctionnant en « double faisceau ».

de rayonnement (lampe deutérium et/ou lampe tungstène-halogène) couplées à un monochromateur à réseau simple ou double ;

- un système optique mettant en forme le faisceau et permettant le fonctionnement en mode « simple faisceau » ou « double faisceau »;

- un dispositif de positionnement de l'objet à mesurer ;

- un système de détection constitué de un ou plusieurs détecteurs (ex. : photomultiplicateur, photodiode au silicium ou InGaAs, détecteur au $\mathrm{PbS}$ ).

La figure 1 présente la disposition des différents éléments pour une configuration de mesure en «double faisceau » en transmission régulière. La séparation du faisceau issu du monochromateur selon deux voies, de référence et de mesure, est réalisée à l'aide d'un miroir tournant repéré « $\mathrm{CBD} »$ sur la figure 1.

La complexité des spectrophotomètres dépend du domaine spectral couvert (nombre de sources et détecteurs) et de l'exactitude requise (monochromateur simple ou double, technique simple ou double faisceau). Les plus performants utilisent un monochromateur double et la technique du double faisceau. Pour des mesures dans le domaine UV, il est recommandé d'utiliser un spectrophotomètre muni d'un monochromateur double à cause des erreurs de mesure dues à la lumière parasite des monochromateurs simples (voir partie 6.10).

La technique du double faisceau fait intervenir simultanément le faisceau de référence (sans échantillon) et le faisceau de mesure (avec échantillon) et permet ainsi de s'affranchir des variations à court terme de l'intensité de la source.

\section{Caractérisation du spectrophotomètre}

Le spectrophotomètre permet de quantifier les propriétés optiques d'un échantillon (transmittance, absorbance, réflectance). Le résultat de la mesure est la valeur de la grandeur associée à la propriété optique (transmission, absorption, réflexion) en fonction de la longueur d'onde. La caractérisation du spectrophotomètre doit donc est faite pour la longueur d'onde et la grandeur optique à mesurer.

La qualification que nous décrivons a été réalisée sur un spectrophotomètre UV-vis.-IR équipé d'un monochromateur double, d'une lampe au deutérium et d'une lampe tungstène-halogène, et fonctionnant en mode double faisceau. Le domaine spectral couvert par le spectrophotomètre s'étend de $250 \mathrm{~nm}$ à $2500 \mathrm{~nm}$. Nous présentons les résultats de la caractérisation obtenus pour les longueurs d'onde de $250 \mathrm{~nm}$ à $800 \mathrm{~nm}$. 


\section{Caractérisation en longueur d'onde}

La mesure des propriétés optiques d'un échantillon nécessite une connaissance des caractéristiques spectrales du faisceau d'analyse. La justesse des valeurs de longueurs d'onde est un paramètre crucial. Les erreurs de mesure dues à un décalage en longueur d'onde ont un impact important sur les valeurs des filtres présentant un fort gradient du facteur de transmission en fonction de la longueur d'onde (filtre coloré). Le décalage en longueur d'onde peut être d'origine mécanique (positionnement du réseau) ou induit par une largeur spectrale inadaptée au filtre à mesurer. La largeur spectrale d'analyse doit être maîtrisée.

\subsection{Justesse spectrale}

La justesse spectrale est vérifiée en mettant en œuvre des techniques qui dépendent du spectrophotomètre. Généralement les spectrophotomètres intègrent des processus d'initialisation mécanique des monochromateurs. Sur l'appareil du LNE, à chaque mise sous tension, l'initialisation de la position des réseaux est réalisée en repérant la raie rouge de la lampe deutérium à $656,1 \mathrm{~nm}$. Pour cela la configuration de mesure en «double faisceau » est désactivée et l'appareil fonctionne en «simple faisceau». Le repérage de la raie rouge est effectué en relevant le maximum du signal mesuré.

La configuration « simple faisceau » est aussi utilisée pour la vérification de la justesse spectrale. Une lampe spectrale à vapeur de mercure mélangée à de l'argon à basse pression est placée dans le compartiment «lampe externe » (fig. 1). Cette lampe émet un spectre de raies fines dont les longueurs d'ondes sont connues avec une incertitude inférieure à 10 pm [4]. On mesure les signaux délivrés par le détecteur du spectrophotomètre en fonction de la longueur d'onde affichée avec un pas d'analyse de $0,01 \mathrm{~nm}$. Les longueurs d'onde correspondant à des maximums de signaux des pics d'émission de la lampe mercure-argon sont comparées aux valeurs de longueurs d'onde d'émission publiées. Cette caractérisation est réalisée en réglant la largeur des fentes de monochromateur pour avoir une largeur spectrale la plus fine possible afin de discriminer les raies d'émission très proches (ex. : $365 \mathrm{~nm}, 577 \mathrm{~nm}$ ). Un exemple de mesure pour une raie du mercure à la longueur d'onde de 546,1 nm avec une largeur spectrale d'analyse de $1 \mathrm{~nm}$ est montré sur la figure 2 .

À partir de ces mesures, il est possible de déterminer les écarts de justesse des valeurs des longueurs d'onde du spectrophotomètre. Si les écarts de justesse sont plus importants que la tolérance établie il est parfois possible de les corriger mécaniquement ou numériquement. La figure 3 montre le résultat obtenu pour les longueurs d'onde entre $200 \mathrm{~nm}$ et $800 \mathrm{~nm}$.

Si une mise en œuvre d'une lampe au mercure dans le compartiment des lampes est impossible une alternative consiste à mesurer le spectre de transmission de filtres de

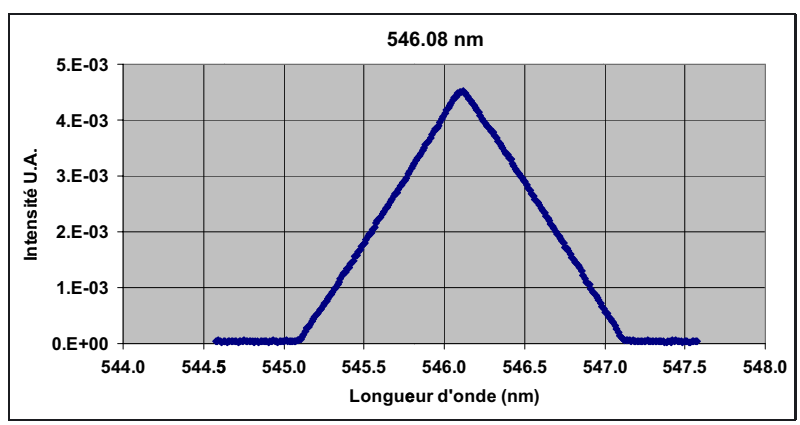

Fig. 2. - Réponse du spectrophotomètre pour la raie du mercure à 546,08 nm.

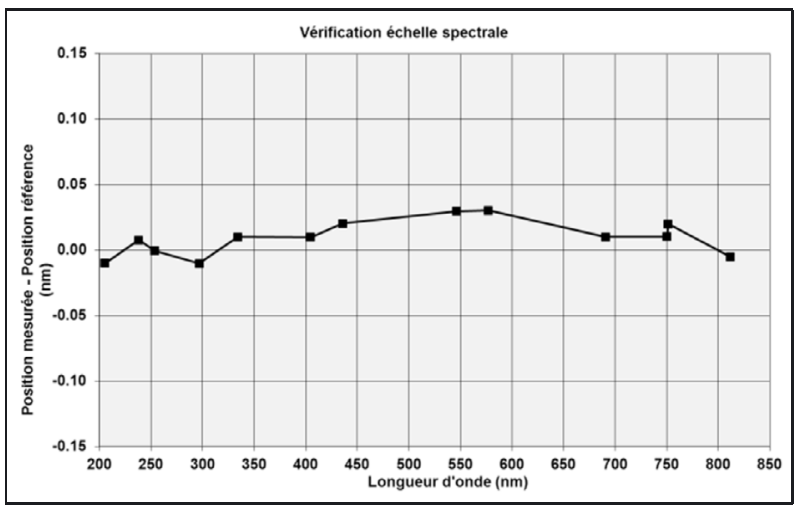

Fig. 3. - Écarts de justesse en longueur d'onde du spectrophotomètre.

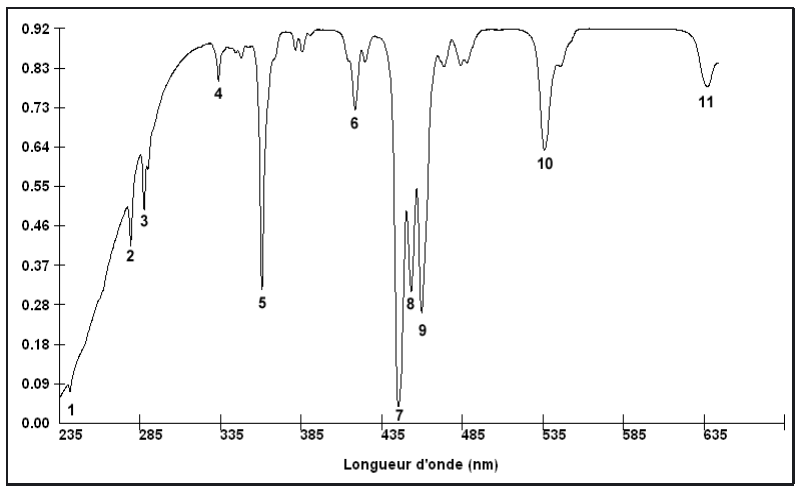

Fig. 4. - Spectre de transmission d'un filtre holmium.

référence tels que les filtres dopés à l'holmium ou au didymium. Ces filtres possèdent des pics d'absorption qui peuvent être étalonnés. Un exemple de spectre de transmission du filtre à l'holmium est présenté sur la figure 4 .

L'incertitude associée à la justesse en longueur d'onde est déterminée par :

- le décalage spectral si la correction en longueur d'onde n'est pas effectuée;

- la répétabilité des mesures du décalage spectral si la correction en longueur d'onde est effectuée;

- l'incertitude d'étalonnage du filtre de référence, si utilisation d'un tel filtre (holmium ou didymium). 


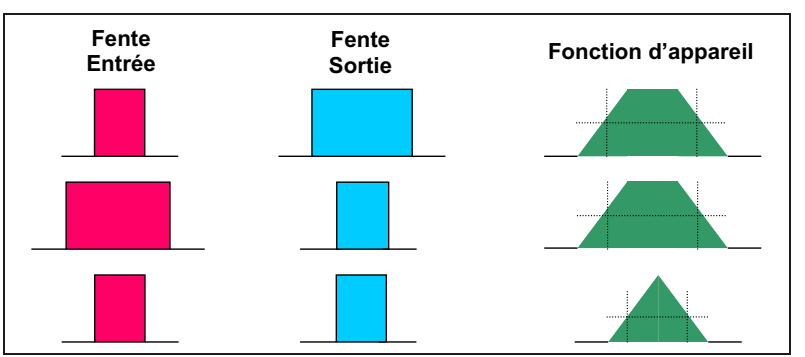

Fig. 5. - Fonction d'appareil d'un monochromateur en fonction de la largeur relative des fentes d'entrée et de sortie.

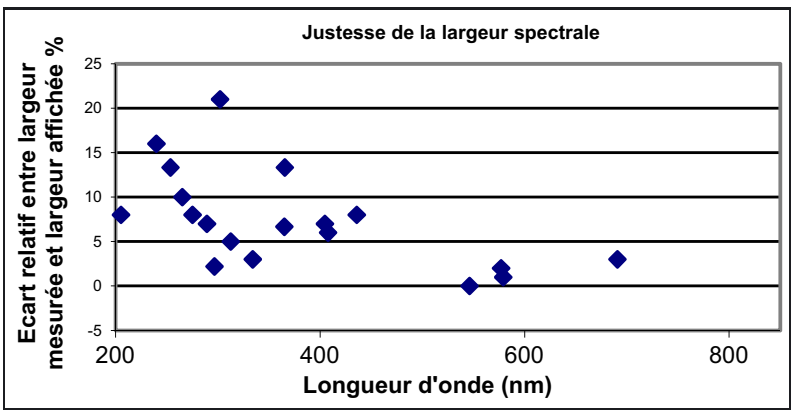

Fig. 6. - Caractérisation de la largeur spectrale d'analyse.

Pour les résultats présentés en figure 3, l'amplitude maximum des décalages en longueur d'onde est de 0,04 nm. L'incertitude type sur les longueurs d'onde entre $200 \mathrm{~nm}$ et $800 \mathrm{~nm}$ est la moitié de l'amplitude maximum, soit $0,02 \mathrm{~nm}$.

\subsection{Justesse des largeurs spectrales}

La largeur spectrale d'analyse du spectrophotomètre dépend des caractéristiques du monochromateur à savoir : les paramètres du réseau utilisé, la longueur de la focale du monochromateur et les largeurs de fentes. Elle caractérise la fonction d'appareil du spectrophotomètre (fig. 5) qui est obtenue en faisant la convolution spatiale des largeurs des fentes d'entrée et de sortie exprimées en millimètre, et en prenant en compte la dispersion spatiale du réseau exprimée en $\mathrm{nm} / \mathrm{mm}$ au niveau de la fente de sortie. Le fonctionnement optimum du spectrophotomètre, en termes de rapport signal à bruit et résolution spectrale, est obtenu lorsque les largeurs des fentes d'entrée et de sortie du monochromateur sont égales.

Les pics d'émission des lampes spectrales (uniquement les raies uniques) sont utilisés pour évaluer l'exactitude de la largeur spectrale d'analyse. Pour chaque pic, les signaux mesurés en fonction de la longueur d'onde sont de forme approximativement triangulaire (fig. 2), si les largeurs des fentes d'entrée et de sortie sont égales. Les longueurs d'onde correspondant à la mi-hauteur des triangles sont relevées afin de calculer les largeurs à mihauteur. La justesse de la largeur spectrale est quantifiée par l'écart entre la largeur à mi-hauteur mesurée et la largeur affichée par le logiciel du spectrophotomètre.

Les résultats de la caractérisation en longueur d'onde de la largeur spectrale d'analyse, pour une largeur de fente donnée, sont présentés sur la figure 6. C'est la

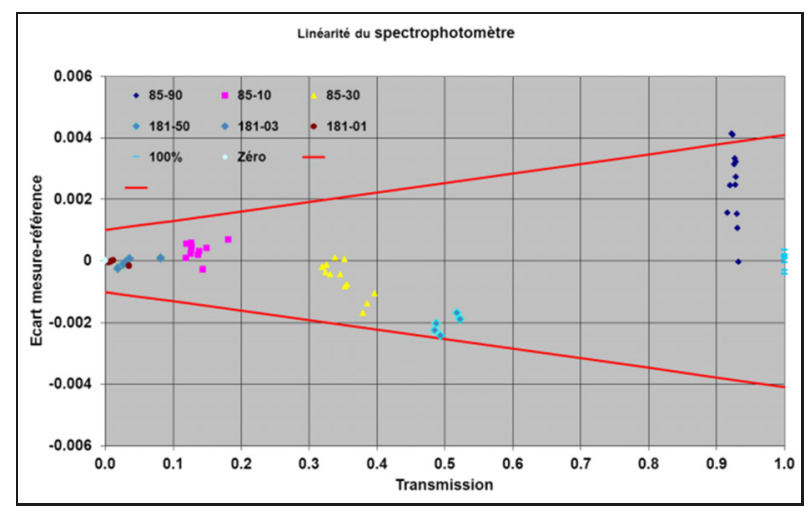

Fig. 7. - Caractérisation de la linéarité du spectrophotomètre.

largeur spectrale mesurée qui doit être prise en compte dans l'évaluation de l'incertitude sur les valeurs de transmission des filtres.

\section{Caractérisation de la linéarité de la réponse du spectrophotomètre}

La mesure précise de la propriété optique d'un échantillon repose sur la linéarité de la réponse du détecteur du spectrophotomètre. Chaque détecteur doit être caractérisé en linéarité. Pour vérifier la linéarité du spectrophotomètre, il faut disposer d'un jeu de filtres étalons de différentes valeurs de transmission, régulièrement étalonnés en facteur de transmission spectrale régulière $[5,6]$. Ces filtres sont étalonnés à l'aide d'un banc de mesure primaire par le LNE-LCM (anciennement dénommé BNMINM ou LNE-INM) [7]. Ces mesures sont traçables au SI [8].

La linéarité est évaluée en calculant les écarts entre des valeurs mesurées par le LNE et les valeurs de références mesurées par le LNE-LCM. La figure 7 présente ces écarts en fonction du facteur de transmission régulier spectral pour les différents domaines spectraux. Les résultats du LNE sont les moyennes de 3 ou 4 séries de mesures. Pour chacun des filtres la dispersion selon l'axe horizontal traduit les variations de la valeur de transmission en fonction de la longueur d'onde.

Les écarts relevés qui traduisent la non-linéarité n'entrainent pas de correction des valeurs mesurées. Ils sont traités comme une composante d'incertitude et sont explicités dans la partie 6 . Les droites de la figure 7 représentent les limites des erreurs de justesse prises en compte pour chaque domaine spectral. La dispersion des points correspondant à des mesures sans filtre (transmission de $100 \%$ ) montrent le niveau de bruit du spectrophotomètre.

\section{6. Évaluation des incertitudes de mesure}

Les incertitudes $(u)$ sont évaluées en appliquant la méthode décrite bans le GUM «Guide pour l'évaluation des incertitudes de mesure » [9]. 


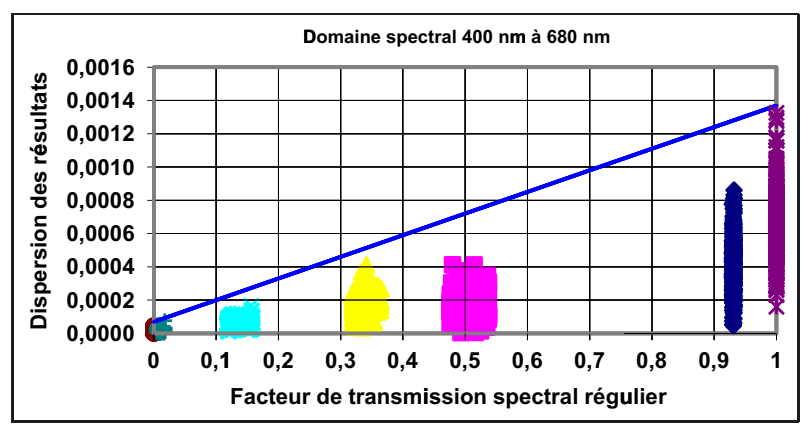

Fig. 8. - Reproductibilité des mesures de transmission.

L'évaluation décrite ci-après s'applique au type de spectrophotomètre que nous avons caractérisé (monochromateur double, double faisceau). Pour d'autres types de spectrophotomètre, il faut prendre en compte les spécificités de l'instrument.

\subsection{Reproductibilité}

La reproductibilité des mesures de transmission est évaluée à partir des résultats de 3 à 4 séries de mesures effectuées à des dates différentes sur un jeu de filtres étalons (85-90, 85-30, 85-10, 181-50, 181-01). Ces mesures sont complétées par :

- une mesure de transmission sans filtre; facteur de transmission égal à 1 ;

- une mesure de transmission en obturant le faisceau; facteur de transmission égal à 0 .

La figure 8 présente les dispersions (maxi - mini) des résultats en fonction du facteur de transmission spectral régulier pour les longueurs d'onde de $400 \mathrm{~nm}$ à $680 \mathrm{~nm}$. L'élargissement horizontal des paquets de points est dû à la variation du facteur de transmission en fonction de la longueur d'onde. Cet élargissement est absent pour une transmission de $100 \%$ (transmission dans l'air). La droite oblique représente les incertitudes élargies dues aux bruits prises en compte pour le domaine spectral considéré.

Les incertitudes types de reproductibilité, $u_{\text {repro, }}$, pour l'ensemble du domaine spectral étudié sont données par les relations (1):

- de $250 \mathrm{~nm}$ à $399 \mathrm{~nm}$ :

$$
u_{\text {repro }}=\left(0,00013+0,001 \tau_{\lambda}\right) / 2
$$

- de 400 nm à 680 nm :

$$
u_{\text {repro }}=\left(0,00004+0,0007 \tau_{\lambda}\right) / 2
$$

- de $681 \mathrm{~nm}$ à $800 \mathrm{~nm}$ :

$$
u_{\text {repro }}=\left(0,00028+0,0009 \tau_{\lambda}\right) / 2
$$

où $\tau_{\lambda}$ est le facteur de transmission spectral.

\subsection{Instabilité temporelle des faisceaux}

L'instabilité temporelle des faisceaux peut être due aux variations de l'intensité de la source et du facteur de transmission de l'air sur les trajets optiques. Dans notre cas, les mesures sont faites en mode « double faisceau ». Les voies de référence et de mesure sont mesurées alternativement avec une fréquence de plusieurs dizaines de hertz et les fluctuations d'intensité sur une durée très courte (inférieure à $0,1 \mathrm{~s}$ ) sont négligeables. Les trajets optiques des deux voies étant sensiblement identiques, ce mode de fonctionnement permet donc de réduire fortement les conséquences des instabilités de la source et des variations de transmission de l'air (les deux voies varient simultanément).

En conséquence, l'incertitude sur le facteur de transmission spectral mesuré due à l'instabilité des faisceaux est considérée comme négligeable et est prise en compte dans l'incertitude de reproductibilité.

\subsection{Incertitude due à la longueur d'onde}

L'incertitude $\left(u_{\lambda}\right)$ due à la longueur d'onde $(\lambda)$ est évaluée à partir de la caractérisation décrite au paragraphe 3. L'incertitude type sur le facteur de transmission spectral mesuré due à l'incertitude $u_{\text {longueur d'onde sur }}$ la longueur d'onde est donnée par la relation (2) :

$$
u_{\lambda}\left(\tau_{\lambda}\right)=\frac{\partial \tau_{\lambda}}{\partial \lambda} u(\lambda)
$$

La dérivée $\frac{\partial \tau_{\lambda}}{\partial \lambda}$ est calculée à partir des points expérimentaux situés de part et d'autre de la longueur d'onde concernée.

Par exemple sur le domaine spectral de $200 \mathrm{~nm}$ à 800 nm (fig. 2), l'incertitude sur la longueur d'onde est de $0,02 \mathrm{~nm}$. Par conséquent l'incertitude sur le facteur de transmission due à la longueur d'onde est :

$$
u_{\lambda}\left(T_{\lambda}\right)=\frac{\partial \tau_{\lambda}}{\partial \lambda} 0,02
$$

où $\frac{\partial \tau_{\lambda}}{\partial \lambda}$ est exprimé en $\mathrm{nm}^{-1}$.

\subsection{Incertitude due à la largeur de la bande spectrale d'analyse}

La distribution spectrale du faisceau transmis par le filtre à étalonner est le produit du spectre du faisceau incident et du facteur de transmission. Le spectre du faisceau incident est défini par la largeur de la bande spectrale d'analyse. Si le filtre présente une variation de la transmission dont la dérivée seconde par rapport à la longueur d'onde est non nulle alors le spectre du faisceau transmis est déformé et la longueur d'onde effective est décalée par rapport à la longueur d'onde sélectionnée.

Par conséquent, l'incertitude due à la largeur spectrale est traitée comme l'incertitude due à la justesse en 


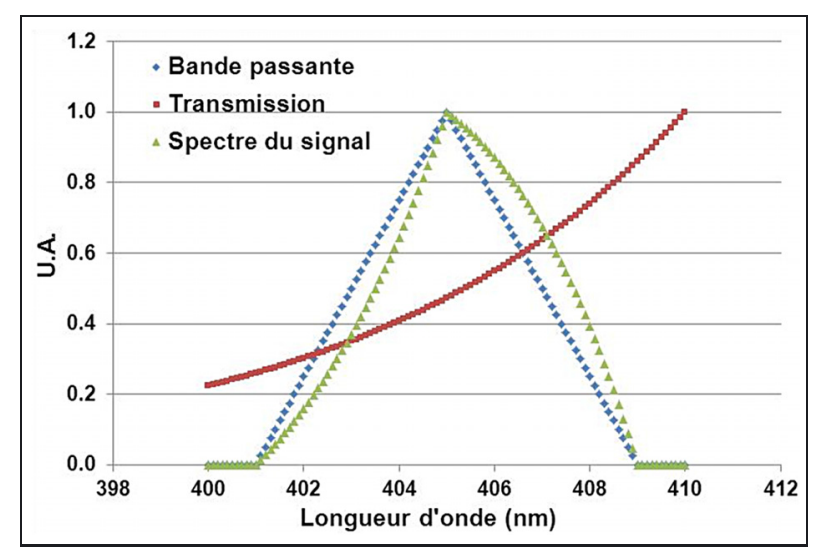

Fig. 9. - Décalage spectral induit par la largeur de bande.

longueur d'onde. Il faut préalablement déterminer le décalage spectral engendré par la largeur spectrale d'analyse. Il peut être obtenu par simulation en prenant comme caractéristique de la largeur spectrale d' analyse une fonction triangulaire correspondant à la fonction de transfert du monochromateur du spectrophotomètre, et le spectre de transmission du filtre. Le produit de ces deux spectres donne le spectre du signal vu par le détecteur, courbe en forme de dent de requin sur la figure 9, dont la longueur d'onde centrale peut être différente de la longueur d'onde choisie (longueur d'onde centrale de la courbe triangulaire).

Une estimation de l'incertitude sur la mesure de transmission due à la largeur spectrale d'analyse peut être obtenue en effectuant des mesures avec des largeurs spectrales d'analyse différentes à condition que le rapport signal sur bruit soit suffisant.

Dans les cas les plus courants (transmission de filtres neutres) l'incertitude due à la largeur spectrale est négligeable. Toutefois ce paramètre d'étalonnage doit être spécifié dans le certificat ou rapport d'étalonnage.

\subsection{Erreur de justesse en transmission}

Les résultats de la caractérisation de la linéarité du spectrophotomètre et donc de la justesse en transmission sont présentés sans la partie 5 de l'article. Les erreurs de justesse si elles ne sont pas corrigées doivent être incluses dans les incertitudes. Sur la figure 6, les droites représentant les valeurs $\Delta_{\text {justesse }}$ sont à prendre en compte pour évaluer l'incertitude due à l'erreur de justesse. Cette incertitude inclut l'incertitude d'étalonnage $u_{\text {étalonnage filtre }}$ des filtres de référence qui sont utilisés pour caractériser le spectrophotomètre et est donnée par l'équation (4).

$$
u_{\text {justesse }}=\sqrt{\left(\frac{\left|\Delta_{\text {justesse }}\right|}{2}\right)^{2}+\left(u_{\text {étalonnage filtre }}\right)^{2}}
$$

Les relations donnant les incertitudes types d'étalonnage prises en compte sont indiquées dans le tableau 1.
Tableau 1

Données pour l'évaluation de l'incertitude due à la justesse.

\begin{tabular}{|c|c|c|c|}
\hline $\begin{array}{c}\text { Longueur } \\
\text { d'onde }\end{array}$ & $\begin{array}{c}\text { Transmission } \\
\text { spectrale }\end{array}$ & $\left|\Delta_{\text {justesse }}\right|$ & $\begin{array}{c}\text { Incertitude } \\
\text { type }\end{array}$ \\
\hline $250 \mathrm{~nm}$ à & $0<\tau_{\lambda}<0,03$ & $0,0003+$ & $0,00015+0,0019 \tau_{\lambda}$ \\
\cline { 2 - 3 } $360 \mathrm{~nm}$ & $0,03<\tau_{\lambda}<1$ & $0,005 \tau_{\lambda}$ & $0,0003+0,0019 \tau_{\lambda}$ \\
\hline \multirow{3}{*}{$360 \mathrm{~nm}$ à } & $0<\tau_{\lambda}<0,03$ & $0,0002+$ & $0,00015+0,0019 \tau_{\lambda}$ \\
\cline { 2 - 3 } $800 \mathrm{~nm}$ & $0,03<\tau_{\lambda}<0,1$ & $0,005 \tau_{\lambda}$ & \multicolumn{2}{|c|}{$0,0003+0,0019 \tau_{\lambda}$} \\
\cline { 2 - 3 } & $0,1<\tau_{\lambda}<1$ & $\begin{array}{c}0,0003+ \\
0,004 \tau_{\lambda}\end{array}$ & \\
\hline
\end{tabular}

\subsection{Incertitude due aux conditions géométriques de mesure}

Les paramètres géométriques pouvant avoir une influence sur le résultat sont :

- la dimension de la surface d'analyse;

- l'angle d'incidence du faisceau par rapport à l'échantillon;

- l'ouverture géométrique du faisceau incident.

Les influences de ces paramètres et les incertitudes associées ne peuvent pas être quantifiées a priori et dépendent principalement de l'objet à étalonner. En conséquence les conditions géométriques de mesures doivent être clairement indiquées avec le résultat de la mesure et en règle générale, l'incertitude due aux paramètres géométriques est supposée nulle.

Cependant il faut être vigilant quant à l'influence de ces paramètres. S'il est prévisible ou constaté (par exemple par des comparaisons de résultats ou de la nature de l'objet) que les conditions géométriques ont une influence non négligeable sur la transmission, une étude paramétrique doit être faite afin de quantifier l'incertitude due aux paramètres géométriques. Cette incertitude due aux paramètres géométriques doit alors être évaluée et intégrée dans l'incertitude globale sur la transmission.

\subsection{Incertitude due au filtre à étalonner}

Les paramètres, autres que les conditions géométriques de mesure, pouvant avoir une influence sur le résultat du fait d'une caractéristique particulière de l'échantillon sont :

- la température de l'échantillon : filtre photochromique, en couches minces (filtres interférentiels, miroirs diélectriques...);

- l'humidité ambiante au niveau de l'échantillon (matériaux hygroscopiques);

- la polarisation du faisceau incident;

- le trajet optique dans l'éprouvette; déviation du faisceau pour les objets optiquement épais ou à faces non-planes;

- la diffusion du rayonnement. 
Tableau 2

Évaluation des composantes d'incertitude.

\begin{tabular}{|c|c|c|c|c|}
\hline & & \multicolumn{3}{|c|}{ Longueur d'onde (nm) } \\
\hline & & 250 à 360 & 360 à 680 & 680 à 800 \\
\hline Sources d'incertitude & $\begin{array}{l}\text { Domaine de } \\
\text { transmission }\end{array}$ & \multicolumn{3}{|c|}{ Valeurs des composantes d'incertitude $u_{\mathrm{x}}\left(\tau_{\lambda}\right)$} \\
\hline $\begin{array}{l}\text { Bruits } \\
\text { (reproductibilité) }\end{array}$ & $0<\tau_{\lambda}<1$ & $0,00013+0,001 \tau_{\lambda}$ & $0,00004+0,0007 \tau_{\lambda}$ & $0,00028+0,0009 \tau_{\lambda}$ \\
\hline \multirow{2}{*}{ Erreur de justesse } & $0<\tau_{\lambda}<0,1$ & \multirow{2}{*}{$0,0003+0,005 \tau_{\lambda}$} & $0,0002+0,005 \tau_{\lambda}$ & $0,0002+0,005 \tau_{\lambda}$ \\
\hline & $0,1<\tau_{\lambda}<1$ & & $0,0003+0,004 \tau_{\lambda}$ & $0,0003+0,004 \tau_{\lambda}$ \\
\hline Filtres étalons & $0<\tau_{\lambda}<1$ & \multicolumn{3}{|c|}{$0,0003+0,0019 \tau_{\lambda}$} \\
\hline Résolution & $0<\tau_{\lambda}<1$ & \multicolumn{3}{|c|}{0,00001} \\
\hline Longueur d'onde & $0<\tau_{\lambda}<1$ & \multicolumn{3}{|c|}{$0,25 d \tau_{\lambda} / d \lambda$} \\
\hline
\end{tabular}

Une caractérisation de ces contributions doit être réalisée pour évaluer leur impact sur la mesure qui se traduit par une correction des valeurs mesurées ou une intégration dans le bilan d'incertitude en fonction de l'importance de cette correction.

\subsection{Conditions environnementales}

Les paramètres environnementaux pouvant avoir une influence sur les résultats sont :

- la température et l'humidité de l'air du laboratoire;

- les vibrations mécaniques de la partie optique du spectrophotomètre;

- Le temps de mise en régime.

Le spectrophotomètre est placé dans un local où la température et l'humidité sont régulées respectivement à $23{ }^{\circ} \mathrm{C} \pm 2{ }^{\circ} \mathrm{C}$ et $50 \% \mathrm{HR} \pm 10 \%$.

Le spectrophotomètre est placé sur une table optique massive. Les incertitudes dues aux vibrations sont supposées nulles sous réserve que le spectrophotomètre et la table ne subissent pas de chocs pendant les mesures. On estime que les incertitudes résiduelles dues aux vibrations sont intégrées dans l'incertitude de reproductibilité des mesures.

\subsection{Résolution}

La résolution des valeurs de transmission est inférieure à $10^{-5}$. L'incertitude due à la résolution est considérée comme négligeable.

\subsection{Lumière parasite}

Le taux de lumière parasite du spectrophotomètre est évalué en mesurant le facteur de transmission de filtres dits «passe-haut». Ce type de filtre est opaque aux courtes longueurs d'onde et présente une forte transmission aux grandes longueurs d'onde. Le taux de lumière parasite est important si la transmission aux courtes longueurs d'onde n'est pas nulle. La figure 10 montre la transmission d'un filtre de type GG455 mesurée à l'aide d'un monochromateur simple. A la longueur d'onde de $350 \mathrm{~nm}$ la transmission mesurée est de 0,095 alors

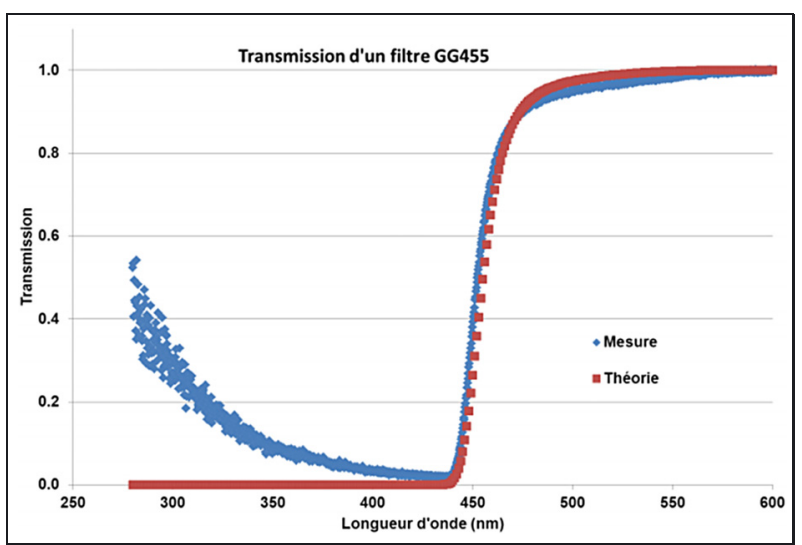

Fig. 10. - Effet de la lumière parasite d'un monochromateur simple sur la transmission d'un filtre «passe-haut».

qu'elle devrait être inférieure à 0,000 01 (sur le domaine spectral $280 \mathrm{~nm}-430 \mathrm{~nm}$ ).

Le spectrophotomètre du LNE intègre un monochromateur double dont le principal intérêt est de présenter un taux de lumière parasite faible. Ceci est confirmé par la mesure de la transmission d'une série de filtres passehaut tels que des filtres de type WG, OG et RG pour le domaine UV et visible. Cette évaluation permet de négliger l'incertitude due à la lumière parasite.

\subsection{Incertitude sur la transmission régulière spectrale}

L'incertitude type composée pour le facteur de transmission spectrale régulière est obtenue en faisant la somme quadratique des composantes d'incertitude évaluées dans les parties 6.1 à 6.10 de l'article. L'incertitude élargie est égale à l'incertitude type composée multipliée par le facteur d'élargissement, $k$, pris généralement égal à 2 .

Pour des applications particulières, la valeur de la composante d'incertitude due au bruit de mesure peut être réduite en répétant sur l'échantillon plusieurs séries de mesures (plusieurs spectres) et en quantifiant l'incertitude type due au bruit de mesure par l'écart type expérimental des valeurs obtenues.

Le tableau 2 présente les diverses composantes d'incertitude quantifiables a priori (non dépendantes de l'échantillon). 


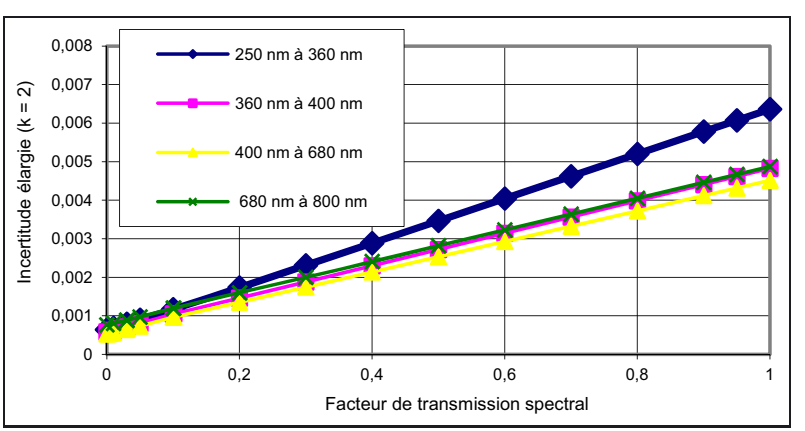

Fig. 11. - Incertitude élargie minimale pour les mesures de transmission régulière spectrale avec le spectrophotomètre Lambda 900.

La figure 11 donne les incertitudes élargies actuelles du LNE pour le facteur de transmission de filtres pour les différentes bandes spectrales.

\section{Conclusion}

La qualification métrologique des spectrophotomètres utilisés pour la caractérisation des matériaux est une opération indispensable pour déterminer les performances de ces instruments. Elle permet de mettre en évidence et d'évaluer des sources d'erreurs telles que la lumière parasite.

La procédure décrite pour réaliser cette qualification permet d'obtenir des informations pour évaluer les incertitudes associées aux deux grandeurs qui forment le résultat d'une mesure à savoir : la longueur d'onde et la propriété optique (transmission, réflexion). Elle peut

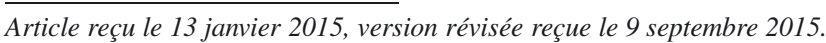

être mise en œuvre dans tous les laboratoires utilisant des spectrophotomètres et des filtres de référence.

\section{Références}

[1] Pfeffer J.C., Skoropinski D.B. et Callis J.B., "Simple, compact visible absorption spectrophotometer", Anal. chem., 56, 14, 1984.

[2] Ren Z., Lin G., Dai L., Huang Z. et Zug L., "Development of a novel spectrophotometer for biochemical analyser based on volume holography transmissive grating and linear CCD”, J. Phys.: Conf. Ser., 277, 2011.

[3] Nevas S., MaNoocheri F. et IKONEN E., "Determination of thin-film parameters from high accuracy measurements of spectral regular transmittance", Metrologia, 40, 2003.

[4] READER J. et CORLISS C.H., "Wavelengths and Transition Probabilities for Atoms and Atomic Ions -Part 1", US Department of Commerce - NBS, déc. 1980.

[5] "Practical methods for the measurement of reflectance and transmittance", Rapport technique de la CIE, 130, Commission international de l'éclairage, 1998.

[6] Gaudemer J. et Voyer J., «Pratique des mesures spectrophotométriques par transmission ", $8^{\text {e }}$ Congrès International de Métrologie, 20-23 oct. 1997, Besançon, France, actes du congrès, pp. 243-246.

[7] Lecollinet P. et BAstie J., « Mesure des facteurs de transmission de filtres optiques au BNM-INM », $8^{\mathrm{e}}$ Congrès international de métrologie, 20-23 oct. 1997, Besançon, France, actes du congrès, pp. 343-348.

[8] Obein G. et Bastie J., "Report on the CCPR Key Comparison K6 Spectral regular transmittance", Base de données du BIPM sur les compraisons clés (KCDB), septembre 2008, http://kcdb.bipm.org/AppendixB/ appbresults/ccpr-k6/ccpr-k6_final_report.pdf.

[9] Guide pour l'expression des incertitudes/Guide to the Expression of Uncertainty in Measurement (GUM), JCGM 100:2008, BIPM, 1re édition, 2008. 